(GAD) antibody titers. The dominant antigen recognized by these antibodies is the GABA-synthesizing enzyme GAD.

Method. Patient X, a 17-year-old Hispanic American female who presented to the Child and Adolescent Psychiatry Clinic with the complaint of ataxia \& aphasia associated with anxiety. Patient was referred by the neurology clinic after they could not establish any organic cause of her ataxia or aphasia. After thorough evaluation at the child psychiatry clinic she was given the diagnosis of anxiety secondary to ataxia and aphasia and r/o Conversion Disorder. She was initiated treatment with sertraline for her anxiety. Her sertraline dose was increased gradually up to $100 \mathrm{mg}$ daily. From the beginning the patient also received counseling \& physical therapy. With these combination of treatments, patient's symptoms did not get any better. Her symptoms actually got worse over time. At this point, the Child Psychiatry Clinic sent a message to the neurology clinic for further evaluation of patient due to her progressive gait and speech impairments.

Results. The neurology clinic saw the patient again and did further testing. The patient was positive for high titers of anti-glutamic acid decarboxylase antibodies (Anti-GAD). At this point, the patient was given the diagnosis of Stiff Person Syndrome. Patient was admitted to the hospital for further management. She was treated with benzodiazepines, IV immune globulin, \& steroid. Soon after discharge from the hospital, the patient was seen at the Child Psychiatric Clinic. The patient's mother reported, after the in-patient treatment, the patient's symptoms improved.

Discussion. It is essential for clinicians to look for neurologic \& other general medical conditions while evaluating a patient with possible conversion disorder. A systematic review of 27 studies found that among 1466 patients initially diagnosed with conversion symptoms, the frequency of misdiagnosis was approximately 4 percent. References. BMJ. 2005;331(7523):989. Epub Oct 13. Childhood onset of stiff-man syndrome. JAMA Neurol. 2013;70(12):1531. J Neurol Neurosurg Psychiatry. 2015 Aug; 86(8):840-8. Epub 2014 Dec 15.

\section{Assessing the Benefit-Risk Ratio of Approved Treatments for Bipolar Depression Using Likelihood to be Helped or Harmed (LHH) Analyses}

\author{
Leslie Citrome, MD, $\mathrm{MPH}^{1}$, Michael Tocco, $\mathrm{PhD}^{2}$, \\ Courtney Zeni, $\mathrm{PhD}^{2}$, Andrei Pikalov, MD, $\mathrm{PhD}^{2}$ and \\ Robert Goldman, PhD $^{2}$
}

${ }^{1}$ New York Medical College, Valhalla, NY, USA, and ${ }^{2}$ Sunovion Pharmaceuticals
Inc., Fort Lee, NJ and Marlborough, MA, USA

\section{Abstract}

Background. Four medications are FDA approved for bipolar depression: lurasidone (LUR), cariprazine (CAR), quetiapine IR \& XR (QUE), and olanzapine-fluoxetine combination (OFC). Indirect comparisons for efficacy using Number Needed to Treat (NNT) and for tolerability using Number Needed to Harm $(\mathrm{NNH})$ can be useful clinical benchmarks to aid treatment decisions. Benefit and risk may also be examined using the Likelihood to be Helped or Harmed (LHH). In this post-hoc analysis, we examined the benefit-risk ratio of the four treatments using LHH.

Method. Individual and pooled monotherapy data from shortterm clinical registration trials of patients with bipolar depression were assessed for LUR, CAR, pooled QUE (300 and $600 \mathrm{mg}$ ), and pooled OFC (considered as monotherapy for this study at fixed doses of $6 / 25,6 / 50,12 / 50 \mathrm{mg}$ ) data. NNT estimates were calculated using the proportions of MADRS responders (defined as $\geq$ $50 \%$ improvement at study endpoint) and MADRS remitters (defined as a score of $\leq 10$ [for LUR and CAR] and $\leq 12$ [for QUE and OFC]) at study endpoint. NNH data were calculated for the proportions of patients who discontinued due to an adverse event (AE) and for individual AEs commonly associated with each treatment. $\mathrm{LHH}$ was calculated as the ratio of $\mathrm{NNH} / \mathrm{NNT}$ to determine the benefit-risk ratio.

Results. The NNT estimates for response vs. placebo were: 5 for both LUR 20-60 mg and 80-120 mg; 10 for both CAR $1.5 \mathrm{mg}$ and $3.0 \mathrm{mg}$; 6 for QUE; and 4 for OFC. The NNTs for remission vs placebo were: 7 for LUR 20-60 mg and 9 for LUR $80-120 \mathrm{mg}$; 10 for CAR $1.5 \mathrm{mg}$ and 13 for CAR $3.0 \mathrm{mg}$; 6 for QUE; and 5 for OFC. The NNH estimates for discontinuations due to AEs were: 642 for LUR 20-60 mg and -151 for LUR 80-120 mg; 298 for CAR $1.5 \mathrm{mg}$ and 31 for CAR $3.0 \mathrm{mg}$; 10 for QUE; and -37 for OFC. NNH values that were negative were assigned a value of 1000 to permit LHH to be calculated. The LHHs for response vs discontinuation due to an AE were: 128.4 for LUR 20-60 mg and 200 for LUR 80-120 mg; 29.8 for CAR $1.5 \mathrm{mg}$ and 3.1 for CAR 3.0 mg; 1.7 for QUE; and 250 for OFC. The LHHs for response vs akathisia were: 3.6 for LUR 20-60 mg and 2.4 for LUR $80-120 \mathrm{mg}$; 3.6 for CAR $1.5 \mathrm{mg}$ and 1.3 for CAR $3.0 \mathrm{mg}$; 34 for QUE; and not available (NA) for OFC. The LHHs for response vs EPS were: 8 for LUR 20-60 mg and 3.2 for LUR 80-120 mg; 5 for CAR $1.5 \mathrm{mg}$ and 2.5 for CAR 3.0 mg; NA for QUE; and NA for OFC. The LHH for response vs weight gain was 5.8 for LUR $20-60 \mathrm{mg}$ and 1110 for LUR 80-120 mg; 5 for both doses of CAR; 2.7 for QUE; and 1.5 for OFC.

Conclusions. LHH can illustrate the trade-offs regarding potential benefits versus potential harms. Across a variety of measures, the lower-dose groups for both LUR and CAR generally evidenced a better benefit-risk profile than the higher-dose groups. While quetiapine and OFC demonstrated robust efficacy, their reduced tolerability resulted in a more marginal benefit-risk ratio for some of the outcomes.

Funding. Sunovion Pharmaceuticals Inc. 